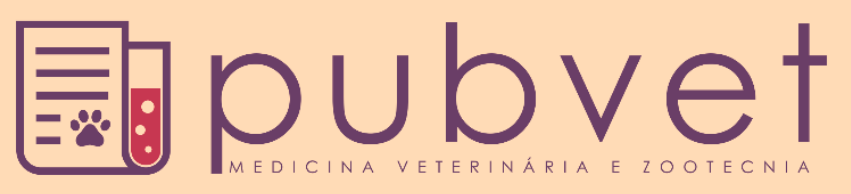

https://doi.org/10.31533/pubvet.v15n04a794.1-8

\title{
Efeito de diferentes soluções de enema sobre variáveis bioquímicas e do equilíbrio ácido-base e eletrolítico de equinos hígidos
}

\author{
Ubiratan Pereira de Melo ${ }^{1 *} \mathbb{G}$, Maristela Silveira Palhares ${ }^{2 \mathbb{9}}$, Cíntia Ferreira ${ }^{10}$, Fabíola \\ Oliveira Paes Leme ${ }^{2 \Phi}$, José Monteiro da Silva Filho ${ }^{2} 9$ \\ ${ }^{l}$ Faculdade Maurício de Nassau (Uninassau) - Campus Capim Macio, Natal-RN, Brasil. E-mail: ubiratan_melo@yahoo.com.br \\ ${ }^{2}$ Escola de Veterinária da UFMG. Belo Horizonte-MG, Brasil. \\ *Autor para correspondência, E-mail: ubiratan_melo@yahoo.com.br
}

Resumo. Este estudo teve por objetivo avaliar o efeito de diferentes soluções de enema sobre os parâmetros bioquímicos, equilíbrio ácido-base e eletrolítico de equinos hígidos. Foram utilizados 15 equinos adultos divididos em três grupos: Grupo 1 - água de torneira associada com sabão neutro; Grupo 2 - solução isotônica; Grupo 3 - água de torneira associada a vaselina. Os efeitos das soluções de enema sobre avaliados variaram em função do tipo de solução infundida. A solução de água de torneira e sabão neutro induziu alcalose metabólica e diminuição da concentração sérica de sódio, cloretos e cálcio total.

Palavras-chave: enema, equino, abdome agudo, equilíbrio ácido-base, eletrólitos

\section{Effect of different enema solutions on biochemical variables and the acid-base and electrolyte balance of healthy horses}

\begin{abstract}
This study aimed to evaluate the effect of different enema solutions on the biochemical parameters, acid-base, and electrolyte balance of healthy horses. Fifteen adult horses were used, divided into three groups: group 1 - treated with tap water associated with neuter soap; group 2 -treated with an isotonic solution; and group 3 - treated with tap water associated with vaseline. The effects of enema solutions on subjects varied according to the type of solution infused. The solution of tap water and neutral soap induced metabolic alkalosis and decreased serum concentration of sodium, chlorides, and total calcium.
\end{abstract}

Keywords: enema, equine, acute abdomen, acid-base balance, electrolytes

\section{Efecto de diferentes soluciones de enemas sobre las variables bioquímicas y el equilibrio ácido-base y electrolítico de caballos sanos}

Resumen. Este estudio tuvo como objetivo evaluar el efecto de diferentes soluciones de enema sobre los parámetros bioquímicos, equilibrio ácido-base y electrolítico de caballos sanos. Se utilizaron quince caballos adultos, divididos en tres grupos: Grupo 1- agua del grifo asociada con jabón neutro; Grupo 2- solución isotónica; Grupo 3- agua del grifo asociada a vaselina. Los efectos de las soluciones de enema en los sujetos variaron según el tipo de solución infundida. La solución de agua del grifo y jabón neutro indujo alcalosis metabólica y disminución de la concentración sérica de sodio, cloruros y calcio total.

Palabras clave: enema, equino, abdomen agudo, equilibrio ácido-base, electrolitos 


\section{Introdução}

A compactação simples é a desordem mais comum do cólon menor dos equinos, ocorrendo secundária ao consumo inadequado de água, ingestão de alimentos fibrosos e de baixa qualidade, alterações odontológicas e da motilidade gastrintestinal (Ferreira et al., 2009; Melo et al., 2007).

As soluções administradas através de enemas hidratam e amolecem as compactações localizadas no cólon menor e reto, além de estimularem a peristalse intestinal (Melo et al., 2008). As soluções de enema composta por água morna e sabão são as mais utilizadas na rotina clínica, e atuam por lubrificação, redução da tensão superficial e amolecimento da compactação (Edwards, 1997). Independente destas ações, pouco se conhece sobre o mecanismo a qual os enemas exercem seus efeitos, existindo duas teorias que explicam seu mecanismo de ação: a teoria do volume e a da irritação química (Schmelzer et al., 2004).

O conteúdo do cólon dos mamíferos é isotônico em relação ao plasma. Desta forma, soluções hipertônicas e hipotônicas infundidas no cólon podem causar alteração hidroeletrolítica, porém soluções isotônicas minimizam tal efeito (Schmelzer et al., 2004). Hiponatremia, hipomagnesemia, hiposmolaridade e hipoferrenemia são complicações comuns em seres humanos após enemas hipotônicos (DiPalma et al., 2003). Repetidos enemas podem causar hipocalemia pela perda excessiva de potássio nas fezes (Jorgensen et al., 1985).

Este estudo teve por objetivo avaliar o efeito de três soluções de enema sobre alguns parâmetros bioquímicos, equilíbrio ácido-base e eletrolítico de equinos hígidos.

\section{Material e Métodos}

Este experimento foi aprovado pelo Comitê de Ética em Experimentação Animal da Universidade Federal de Minas Gerais (CETEA/UFMG) sob o número 19/2006.

Foram utilizados 15 equinos adultos hígidos (12 fêmeas, 3 machos) com peso médio de $262 \pm 48,28$ $\mathrm{kg}$, em um delineamento inteiramente casualizado. Antes do período experimental os animais passaram por período de adaptação de 30 dias durante o qual receberam ração comercial $(0,5 \mathrm{~kg} / 100 \mathrm{~kg}$ P.V.), feno de tifton (Cynodon ssp.) (1,0 kg/100 kg), capim elefante (Pennisetum purpureum) triturado, água e sal mineral ad libitum.

Os animais foram agrupados e divididos em três grupos (4 fêmeas e 1 macho/grupo): Grupo 1 (G1) - água de torneira associada com sabão neutro (seis gramas de sabão neutro/litro de água; osmolaridade: $24 \mathrm{mOsm} / \mathrm{L}$ ); Grupo 2 (G2) - solução eletrolítica (Solução: $\mathrm{Na}^{+}: 135 \mathrm{mEq} / \mathrm{L} ; \mathrm{HCO}_{3}^{-}: 25 \mathrm{mEq} / \mathrm{L}^{-} \mathrm{Cl}^{-}$: $110 \mathrm{mEq} / \mathrm{L} ; \mathrm{K}^{+}: 4 \mathrm{mEq} / \mathrm{L} ; \mathrm{Ca}^{++}: 5 \mathrm{mEq} / \mathrm{L} ;$ osmolaridade: $\left.285 \mathrm{mOsm} / \mathrm{L} ; \mathrm{pH}: 7,45\right)$; Grupo 3 (G3) - água de torneira associada a vaselina líquida (3 litros de água/litro de vaselina; osmolalidade: $6 \mathrm{mOsm} / \mathrm{L}$ ). Foram realizados três enemas em cada animal, sendo um a cada três horas. $\mathrm{O}$ enema foi administrado por meio de fluxo de gravidade em volume equivalente a quatro litros de solução à temperatura de $35^{\circ} \mathrm{C}$. Após cada enema, os animais foram estimulados a caminhar ao passo durante 15 minutos e então a cada hora.

Amostras de sangue foram obtidas por venopunção central na jugular externa, em tubos à vácuo, sem anticoagulante. Após retração do coágulo, o sangue foi centrifugado a $2.000 \mathrm{rpm}$ por cinco minutos, o soro separado em alíquotas de $1,5 \mathrm{~mL}$ e congelado a $-20^{\circ} \mathrm{C}$ até o processamento das análises. A bioquímica do sangue incluiu a mensuração da concentração sérica de creatinina, ureia, proteína total, albumina e glicose, por meio de kits específicos, em aparelho automático de bioquímica. Para o cálculo da osmolaridade plasmática foi adotada a fórmula descrita na literatura (Geovú et al., 2006).

Para determinação do equilíbrio ácido-base e eletrolítico foram colhidas amostras de sangue por venopunção central na jugular externa em seringas de $5 \mathrm{~mL}$ sem anticoagulante. A determinação do equilíbrio acidobásico ( $\mathrm{pH}, \mathrm{TCO}_{2}, \mathrm{PaCO}_{2}$, desvio de base, $\mathrm{HCO}_{3}$, ânion gap), além da determinação da concentração sérica de $\mathrm{Na}^{+}, \mathrm{Cl}^{-}, \mathrm{K}^{+}$foi realizada em analisador clínico portátil utilizando cartuchos específicos. A concentração sérica de $\mathrm{Ca}^{++}$e $\mathrm{Mg}^{++}$foi determinada por meio de kits específicos em aparelho automático de bioquímica. 
As amostras foram colhidas imediatamente antes do primeiro enema $\left(\mathrm{T}_{0}\right)$; uma hora antes do segundo enema $\left(\mathrm{T}_{1}\right)$ e uma hora após o terceiro enema $\left(\mathrm{T}_{2}\right)$.

$\mathrm{O}$ delineamento experimental foi inteiramente ao acaso. O modelo experimental constituiu-se de parcelas subdivididas (três grupos nas parcelas e três tempos nas subparcelas - bioquímica do sangue, gasometria e perfil eletrolítico) com cinco repetições. A análise de variância foi utilizada (ANOVA), considerando-se a ocorrência dos erros (a) e (b), referentes à parcela e subparcela, respectivamente. $\mathrm{O}$ teste estatístico, para comparação das médias, foi o SNK (Student-Newman Keuls) com nível de significância de 95\% ( $\mathrm{P}<0,05)$. Os dados foram analisados pelo programa Statistical Analyses System (SAS, 2004).

\section{Resultados}

A concentração sérica de albumina (Tabela 1) não apresentou diferença $(P>0,05)$ entre os tempos dentro dos grupos 1 e 3 . No grupo 2 houve elevação dos valores séricos, onde $T_{0}$ e $T_{2}$ apresentaram diferença $(P<0,05)$. Não houve diferença $(P>0,05)$ entre os grupos dentro dos tempos. A concentração sérica de proteína não diferiu $(\mathrm{P}>0,05)$ entre os tempos dentro dos grupos 1 e 3 , embora tenha ocorrido elevação dos valores dentro dos grupos $(\mathrm{P}>0,05)$. Já no grupo 2 houve diferença $(\mathrm{P}<0,05)$ entre os tempos, com maiores valores sendo observados no $\mathrm{T}_{2}$ em relação ao $\mathrm{T}_{0}(\mathrm{P}<0,05)$.

A osmolalidade plasmática manteve-se dentro dos limites de referência, não havendo diferença ( $\mathrm{P}$ $>0,05)$ entre os tempos dentro dos grupos 1 e 3 . No grupo 2 , houve elevação dos valores $(P<0,05)$ entre $\mathrm{T}_{0}$ e $\mathrm{T}_{2}$. Não houve diferença $(\mathrm{P}>0,05)$ entre os grupos em $\mathrm{T}_{1}$ e $\mathrm{T}_{2}$, entretanto, observaram-se diferenças $(P<0,05)$ entre os grupos dentro de $T_{2}(G 2>G 1=G 3)$.

Tabela 1. Efeito de diferentes soluções de enema sobre os valores séricos de albumina, proteína total, osmolalidade, uréia, creatinina e glicose de equinos hígidos.

\begin{tabular}{|c|c|c|c|}
\hline \multirow{2}{*}{ Tempos } & \multicolumn{3}{|c|}{ Grupos experimentais } \\
\hline & Água e sabão & Solução eletrolítica & Água e vaselina \\
\hline \multicolumn{4}{|c|}{$\begin{array}{ll} & \text { Albumina } \\
\end{array}$} \\
\hline $\mathrm{T}_{0}$ (antes) & $2,60 \pm 0,92^{\mathrm{Aa}}$ & $2,36 \pm 0,81^{\mathrm{Ba}}$ & $2,60 \pm 0,27^{\mathrm{Aa}}$ \\
\hline $\mathrm{T}_{1}$ (120 min.) & $2,54 \pm 0,61^{\mathrm{Aa}}$ & $2,70 \pm 0,61^{\mathrm{ABa}}$ & $2,62 \pm 0,31^{\mathrm{Aa}}$ \\
\hline $\mathrm{T}_{2}$ (410 min.) & $2,82+0,55^{\text {Aa }}$ & $2,86+0,36^{\mathrm{Aa}}$ & $2,54+0,39^{\mathrm{Aa}}$ \\
\hline \multicolumn{4}{|c|}{$\checkmark \quad$ Proteína total $(6,5-7,5 \mathrm{mg} / \mathrm{dL})$} \\
\hline $\mathrm{T}_{0}$ (antes) & $6,96 \pm 0,11^{\mathrm{Aa}}$ & $6,68 \pm 0,38^{\mathrm{Bb}}$ & $7,02 \pm 0,50^{\mathrm{Aa}}$ \\
\hline $\mathrm{T}_{1}(120$ min. $)$ & $6,90 \pm 0,29^{\mathrm{Aa}}$ & $6,82 \pm 0,43^{\mathrm{ABa}}$ & $6,96 \pm 0,57^{\mathrm{Aa}}$ \\
\hline $\mathrm{T}_{2}(410 \mathrm{~min})$. & $7,00 \pm 0,53^{\mathrm{Ab}}$ & $6,98 \pm 0,44^{\mathrm{Ab}}$ & $7,20 \pm 0,50^{\mathrm{Aa}}$ \\
\hline \multicolumn{4}{|c|}{$\checkmark \quad$ Osmolalidade $(270-300 \mathrm{mOsm} / \mathrm{kg})$} \\
\hline $\mathrm{T}_{0}$ (antes) & $279,80 \pm 4,93^{\mathrm{Aa}}$ & $276,12 \pm 2,58^{\mathrm{Aa}}$ & $277,64 \pm 2,77^{\mathrm{Aa}}$ \\
\hline $\mathrm{T}_{1}$ (120 min.) & $278,23 \pm 4,81^{\mathrm{Aa}}$ & $278,75 \pm 2,74^{\mathrm{Aa}}$ & $277,17 \pm 2,63^{\mathrm{Aa}}$ \\
\hline $\mathrm{T}_{2}(410 \mathrm{~min})$. & $277,08 \pm 5,58^{\mathrm{Ab}}$ & $292,76 \pm 30,1^{\mathrm{Ba}}$ & $277,31 \pm 2,54^{\mathrm{Ab}}$ \\
\hline \multicolumn{4}{|c|}{$\checkmark \quad$ Ureia $(11-26 \mathrm{mg} / \mathrm{dL})$} \\
\hline $\mathrm{T}_{0}$ (antes) & $15,80 \pm 2,28^{\mathrm{Ab}}$ & $17,60 \pm 4,03^{\mathrm{Aa}}$ & $14,00 \pm 1,22^{\mathrm{Ac}}$ \\
\hline $\mathrm{T}_{1}$ (120 min.) & $15,80 \pm 1,65^{\mathrm{Ab}}$ & $17,60 \pm 3,84^{\mathrm{Aa}}$ & $14,40 \pm 1,14^{\mathrm{Ac}}$ \\
\hline $\mathrm{T}_{2}$ (410 min.) & $16,00 \pm 2,00^{\mathrm{Aa}}$ & $18,40 \pm 3,36^{\mathrm{Aa}}$ & $15,00 \pm 0,70^{\mathrm{Aa}}$ \\
\hline \multicolumn{4}{|c|}{$\checkmark \quad$ Creatinina $(0,8-1,8 \mathrm{mg} / \mathrm{dL})$} \\
\hline $\mathrm{T}_{0}$ (antes) & $0,86 \pm 0,11^{\mathrm{Ab}}$ & $1,22 \pm 0,49^{\mathrm{Aa}}$ & $0,84 \pm 0,23^{\mathrm{Ab}}$ \\
\hline $\mathrm{T}_{1}(120 \mathrm{~min})$. & $0,94 \pm 0,15^{\mathrm{Ab}}$ & $1,28 \pm 0,54^{\mathrm{Aa}}$ & $1,02 \pm 0,23^{\mathrm{Ab}}$ \\
\hline $\mathrm{T}_{2}$ (410 min.) & $0,94+0,19^{\mathrm{Ab}}$ & $1,36+0,52^{\mathrm{Aa}}$ & $0,98+0,27^{\mathrm{Ab}}$ \\
\hline \multicolumn{4}{|c|}{$\checkmark \quad$ Glicose $(76-119 \mathrm{mg} / \mathrm{dL})$} \\
\hline $\mathrm{T}_{0}$ (antes) & $90,40 \pm 12,91^{\mathrm{Aa}}$ & $84,20 \pm 2,94^{\mathrm{Ab}}$ & $84,60 \pm 6,06^{\mathrm{Ab}}$ \\
\hline $\mathrm{T}_{1}$ (120 min.) & $87,60 \pm 13,50^{\mathrm{ABa}}$ & $78,60 \pm 5,94^{\mathrm{Bb}}$ & $86,80 \pm 8,16^{\mathrm{Aa}}$ \\
\hline $\mathrm{T}_{2}$ (410 min.) & $83,80 \pm 19,67^{\mathrm{Bab}}$ & $79,20 \pm 7,98^{\mathrm{Bb}}$ & $86,80 \pm 9,03^{\mathrm{Aa}}$ \\
\hline
\end{tabular}

${ }_{\mathrm{AB}}$ Letras diferentes, dentro da coluna, diferem entre si $(\mathrm{P}<0,05)$.

${ }^{\mathrm{ab}}$ Letras diferentes, dentro da linha, diferem entre si $(\mathrm{P}<0,05)$.

A ureia manteve-se dentro dos limites de referência durante o experimento, porém houve diferença $(\mathrm{P}>0,05)$ entre os grupos dentro de $\mathrm{T}_{0}(\mathrm{G} 2>\mathrm{G} 1=\mathrm{G} 3), \mathrm{T}_{1}(\mathrm{G} 3<\mathrm{G} 1<\mathrm{G} 2)$ e $\mathrm{T}_{2}(\mathrm{G} 2>\mathrm{G} 1=\mathrm{G} 3)$. A creatinina manteve-se dentro dos valores de referência, não ocorrendo diferenças $(\mathrm{P}>0,05)$ entre os tempos dentro dos grupos. Em todos os três tempos, houve diferença $(P<0,05)$ entre os grupos $(\mathrm{G} 2>\mathrm{G} 1=\mathrm{G} 3)$. 
A concentração sérica de $\mathrm{Na}^{+}$se manteve dentro dos limites fisiológicos nos três grupos em todos os tempos (Tabela 2). Entretanto, nos grupos $1\left(\mathrm{~T}_{2}\right)$ e $3\left(\mathrm{~T}_{1}\right)$ houve diminuição da concentração sérica deste eletrólito. No grupo 1 ocorreu diferença $(\mathrm{P}<0,05)$ entre $\mathrm{T}_{0}$ e $\mathrm{T}_{2}$.

A concentração sérica de potássio se manteve dentro dos limites de referência para a espécie durante todo o período experimental nos três grupos. Todavia, foi observada discreta diminuição dos valores séricos deste eletrólito em todos os grupos.

A concentração sérica de cloretos manteve-se dentro da faixa de normalidade em todos os grupos, não havendo diferença $(\mathrm{P}>0,05)$ entre os grupos dentro de cada tempo e entre os tempos dentro dos grupos 2 e 3. Entretanto foi observada diferença $(\mathrm{P}<0,05)$ entre o tempo 0 e os tempos 1 e 2 no grupo 1. Observa-se que embora a concentração de cloretos tenha diminuído em todos os grupos, a maior diferença foi observada no grupo 1 (água e sabão).

Tabela 2. Efeito de diferentes soluções de enema sobre os eletrólitos de equinos hígidos.

\begin{tabular}{|c|c|c|c|}
\hline \multirow[b]{2}{*}{ Tempos } & \multicolumn{3}{|c|}{ Grupos experimentais } \\
\hline & Água e sabão & Solução eletrolítica & Água e vaselina \\
\hline \multicolumn{4}{|c|}{$\checkmark \quad$ Sódio (135-145 mEq/L) } \\
\hline $\mathrm{T}_{0}$ (antes) & $135,80 \pm 2,38^{\mathrm{Aa}}$ & $135,60 \pm 1,51^{\mathrm{Aa}}$ & $135,00 \pm 1,58^{\mathrm{Aa}}$ \\
\hline $\mathrm{T}_{1}(120 \mathrm{~min})$. & $135,20 \pm 2,28^{\mathrm{ABa}}$ & $135,40 \pm 1,14^{\mathrm{Aa}}$ & $134,80 \pm 1,30^{\mathrm{Aa}}$ \\
\hline $\mathrm{T}_{2}(410 \mathrm{~min})$. & $134,60 \pm 2,70^{\mathrm{Ba}}$ & $135,60 \pm 1,34^{\mathrm{Aa}}$ & $135,00 \pm 1,64^{\mathrm{Aa}}$ \\
\hline \multicolumn{4}{|c|}{$\checkmark \quad$ Potássio $(3-5 \mathrm{mEq} / \mathrm{L})$} \\
\hline $\mathrm{T}_{0}$ (antes) & $4,06 \pm 0,40^{\mathrm{Aa}}$ & $4,16 \pm 0,26^{\mathrm{Aa}}$ & $4,22 \pm 0,14^{\mathrm{Aa}}$ \\
\hline $\mathrm{T}_{1}$ (120 min.) & $3,90 \pm 0,22^{\mathrm{Aa}}$ & $3,90 \pm 0,33^{\mathrm{Ba}}$ & $4,02 \pm 0,16^{\mathrm{Aa}}$ \\
\hline $\mathrm{T}_{2}(410 \mathrm{~min})$. & $3,96 \pm 0,35^{\text {Аа }}$ & $3,86 \pm 0,38^{\mathrm{Ba}}$ & $3,98 \pm 0,24^{\mathrm{Ba}}$ \\
\hline \multicolumn{4}{|c|}{$\checkmark \quad$ Cloretos $(99-105 \mathrm{mEq} / \mathrm{L})$} \\
\hline $\mathrm{T}_{0}$ (antes) & $103,20 \pm 1,78^{\mathrm{Aa}}$ & $102,40 \pm 1,51^{\mathrm{Aa}}$ & $102,00 \pm 1,87^{\mathrm{Aa}}$ \\
\hline $\mathrm{T}_{1}(120 \mathrm{~min})$. & $101,80 \pm 2,58^{\mathrm{Ba}}$ & $101,60 \pm 2,88^{\mathrm{Aa}}$ & $101,40 \pm 1,67^{\mathrm{Aa}}$ \\
\hline $\mathrm{T}_{2}(410 \mathrm{~min})$. & $101,00 \pm 2,44^{\mathrm{Ba}}$ & $101,80+3,27^{\mathrm{Aa}}$ & $101,40 \pm 1,14^{\mathrm{Aa}}$ \\
\hline \multicolumn{4}{|c|}{$\checkmark \quad$ Cálcio total $(9,6-13,2 \mathrm{mg} / \mathrm{dL})$} \\
\hline $\mathrm{T}_{0}$ (antes) & $10,54 \pm 2,17^{\mathrm{Aa}}$ & $10,30 \pm 2,14^{\mathrm{Aa}}$ & $9,32 \pm 0,90^{\mathrm{Aa}}$ \\
\hline $\mathrm{T}_{1}$ (120 min.) & $9,74+1,15^{\mathrm{Aa}}$ & $9,98+2,31^{\mathrm{Aa}}$ & $8,94 \pm 2,57^{\mathrm{Aa}}$ \\
\hline $\mathrm{T}_{2}$ (410 min.) & $8,82 \pm 0,47^{\mathrm{Aa}}$ & $9,30 \pm 1,73^{\mathrm{Aa}}$ & $9,84 \pm 1,16^{\mathrm{Aa}}$ \\
\hline \multicolumn{4}{|c|}{$\begin{array}{ll}\checkmark \quad \text { Magnésio }(1,4-2,5 \mathrm{mg} / \mathrm{dL}) \\
\end{array}$} \\
\hline $\mathrm{T}_{0}$ (antes) & $1,42 \pm 0,27^{\mathrm{Aa}}$ & $1,90 \pm 0,79^{\mathrm{Aa}}$ & $1,44 \pm 0,27^{\mathrm{Aa}}$ \\
\hline $\mathrm{T}_{1}(120 \mathrm{~min})$. & $1,44 \pm 0,34^{\mathrm{Aa}}$ & $1,60 \pm 0,46^{\mathrm{Aa}}$ & $1,42 \pm 0,58^{\mathrm{Aa}}$ \\
\hline $\mathrm{T}_{2}(410 \mathrm{~min})$. & $1,48 \pm 0,50^{\mathrm{Aa}}$ & $1,86 \pm 0,54^{\mathrm{Aa}}$ & $1,82 \pm 0,54^{\mathrm{Aa}}$ \\
\hline
\end{tabular}

Não houve diferença $(\mathrm{P}>0,05)$ na concentração de cálcio total entre os tempos dentro de cada grupo e entre os grupos dentro de cada tempo. A solução de água com sabão provocou uma diminuição mais marcante na concentração de cálcio total quando comparada às outras soluções. O magnésio se manteve dentro dos limites fisiológicos e não houve diferença $(P>0,05)$ entre os tempos ou grupos.

A tensão de dióxido de carbono $\left(\mathrm{TCO}_{2}\right)$ (Tabela 3) apresentou comportamento diferente entre os grupos. No grupo 1 houve discreta elevação dos seus valores sem, contudo, provocar diferença $(\mathrm{P}>$ $0,05)$ entre os tempos. Já nos grupos 2 e 3 houve diminuição dos valores de $\mathrm{TCO}_{2}$, com diferença $(\mathrm{P}<$ 0,05 ) entre os tempos dentro dos grupos. $\mathrm{A} \mathrm{pCO}_{2}$ (Tabela 3 ) apresentou diminuição dos valores ao longo do tempo.

Seguindo a mesma variação da $\mathrm{TCO}_{2}$ e $\mathrm{pCO}_{2}$, os valores de $\mathrm{pH}$ apresentaram comportamentos diferentes entre os grupos. No grupo 1 houve aumento $(\mathrm{P}<0,05)$ do valor do $\mathrm{pH}$ o que provocou diferença $(\mathrm{P}<0,05)$ entre os tempos 0 e 2 . Diferentemente do grupo 1 , o $\mathrm{pH}$ do grupo 2 se manteve constante durante todo o período experimental não havendo diferença $(\mathrm{P}>0,05)$ entre os tempos dentro deste grupo. Já no grupo 3 foi observada a mesma resposta daquela descrita para o grupo 1 .

Em relação ao bicarbonato $\left(\mathrm{HCO}_{3}{ }^{-}\right)$, o grupo 1 apresentou elevação dos valores ao longo do tempo, sem, contudo, haver diferença $(\mathrm{P}>0,05)$ entre os tempos. Por outro lado, houve uma diminuição dos valores de $\mathrm{HCO}_{3}{ }^{-}$durante os tempos nos grupos 2 e 3 . Todavia a flutuação dos valores no grupo 2 
$\left(\mathrm{T}_{0}>\mathrm{T}_{1}=\mathrm{T}_{2}\right)$ e grupo $3\left(\mathrm{~T}_{0}=\mathrm{T}_{1}>\mathrm{T}_{2}\right)$ provocaram diferença $(\mathrm{P}<0,05)$. A comparação dos grupos dentro de cada tempo revelou diferenças $(\mathrm{P}<0,05)$ nos tempos $0(\mathrm{G} 2=\mathrm{G} 3>\mathrm{G} 1)$ e $2(\mathrm{G} 1=\mathrm{G} 2>\mathrm{G} 3)$.

Embora não significativo $(P>0,05)$ houve elevação do valor do desvio de base no grupo 1 . Já nos grupos 2 e 3 houve diminuição dos valores do desvio de base. $\mathrm{O}$ ânion gap apresentou aumento dos valores dentro dos grupos ao longo dos tempos. No grupo 1, a elevação do ânion gap não foi significativa $(\mathrm{P}>0,05)$. Já nos grupos 2 e 3 houve diferença $(\mathrm{P}<0,05)$ entre os tempos. Já os grupos diferiram $(\mathrm{P}<$ $0,05)$ em $\mathrm{T}_{0}(\mathrm{G} 1>\mathrm{G} 2$; G1 e G2=G3).

Tabela 3. Efeito de diferentes soluções de enema sobre o $\mathrm{TCO}_{2}, \mathrm{PaCO}_{2}, \mathrm{pH}, \mathrm{NaHCO}_{3}$, desvio de base e anion gap de equinos hígidos.

\begin{tabular}{|c|c|c|c|}
\hline \multirow[b]{2}{*}{ Tempos } & \multicolumn{3}{|c|}{ Grupos experimentais } \\
\hline & $\begin{array}{c}\text { Água e sabão } \\
\mathrm{G}_{1} \\
\end{array}$ & $\begin{array}{c}\text { Solução eletrolítica } \\
\mathrm{G}_{2}\end{array}$ & $\begin{array}{c}\text { Água e vaselina } \\
\mathrm{G}_{3} \\
\end{array}$ \\
\hline \multicolumn{4}{|c|}{$\begin{array}{ll} & \text { TCO }_{2}(25-32 \mathrm{mEq} / \mathrm{L}) \\
\end{array}$} \\
\hline $\mathrm{T}_{0}$ (antes) & $29,20 \pm 1,78^{\mathrm{Ab}}$ & $31,60 \pm 2,07^{\mathrm{Aa}}$ & $30,80 \pm 2,04^{\mathrm{Aa}}$ \\
\hline $\mathrm{T}_{1}$ (120 min.) & $29,40 \pm 2,50^{\mathrm{Aa}}$ & $30,40 \pm 2,88^{\mathrm{Ba}}$ & $30,00 \pm 1,73^{\mathrm{Aa}}$ \\
\hline $\mathrm{T}_{2}$ (410 min.) & $29,60 \pm 1,34^{\mathrm{Aa}}$ & $29,80 \pm 2,86^{\mathrm{Ba}}$ & $28,40 \pm 1,14^{\mathrm{Bb}}$ \\
\hline \multicolumn{4}{|l|}{$\begin{array}{ll}\checkmark & \mathrm{PaCO}_{2} \\
\end{array}$} \\
\hline $\mathrm{T}_{0}$ (antes) & $42,64 \pm 2,27^{\mathrm{Aa}}$ & $44,16 \pm 3,36^{\mathrm{Aa}}$ & $44,14 \pm 2,45^{\mathrm{Aa}}$ \\
\hline $\mathrm{T}_{1}(120 \mathrm{~min})$. & $42,40 \pm 2,91^{\mathrm{Aa}}$ & $41,68 \pm 3,04^{\mathrm{Ba}}$ & $42,82 \pm 1,96^{\mathrm{ABa}}$ \\
\hline $\mathrm{T}_{2}$ (410 min.) & $40,46 \pm 1,74^{\mathrm{Ba}}$ & $42,32 \pm 3,40^{\mathrm{ABa}}$ & $41,86 \pm 1,62^{\mathrm{Ba}}$ \\
\hline \multicolumn{4}{|c|}{$\begin{array}{ll}\mathbf{p H}(7,35-7,45) \\
\end{array}$} \\
\hline $\mathrm{T}_{0}$ (antes) & $7,42 \pm 0,01^{\mathrm{Ba}}$ & $7,44 \pm 0,00^{\mathrm{Ab}}$ & $7,43 \pm 0,01^{\mathrm{Aa}}$ \\
\hline $\mathrm{T}_{1}(120 \mathrm{~min})$. & $7,42 \pm 0,02^{\mathrm{Ba}}$ & $7,45 \pm 0,02^{\mathrm{Ab}}$ & $7,43 \pm 0,01^{\mathrm{Aa}}$ \\
\hline $\mathrm{T}_{2}$ (410 min.) & $7,47 \pm 0,01^{\mathrm{Aa}}$ & $7,43 \pm 0,02^{\mathrm{Ab}}$ & $7,39 \pm 0,00^{\mathrm{Ba}}$ \\
\hline \multicolumn{4}{|c|}{ 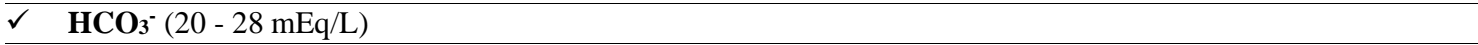 } \\
\hline $\mathrm{T}_{0}$ (antes) & $28,00 \pm 1,78^{\mathrm{Aa}}$ & $30,32 \pm 2,07^{\mathrm{Ab}}$ & $29,56 \pm 2,10^{\mathrm{Ab}}$ \\
\hline $\mathrm{T}_{1}(120 \mathrm{~min})$. & $28,10 \pm 2,62^{\mathrm{Aa}}$ & $29,10 \pm 2,68^{\mathrm{Ba}}$ & $28,82 \pm 1,51^{\mathrm{Aa}}$ \\
\hline $\mathrm{T}_{2}$ (410 min.) & $28,50 \pm 1,18^{\mathrm{Aa}}$ & $28,76 \pm 3,08^{\mathrm{Ba}}$ & $27,18 \pm 1,40^{\mathrm{Bb}}$ \\
\hline \multicolumn{4}{|c|}{$\checkmark \quad$ Desvio de base $(-4-+4)$} \\
\hline $\mathrm{T}_{0}$ (antes) & $3,60 \pm 2,19^{\mathrm{Aa}}$ & $6,20 \pm 1,78^{\mathrm{Ab}}$ & $5,60 \pm 2,19^{\mathrm{Ab}}$ \\
\hline $\mathrm{T}_{1}(120 \mathrm{~min})$. & $3,80 \pm 2,86^{\mathrm{Ab}}$ & $5,40 \pm 2,88^{\mathrm{ABa}}$ & $4,80 \pm 1,48^{\mathrm{Aab}}$ \\
\hline $\mathrm{T}_{2}(410 \mathrm{~min})$. & $4,60 \pm 1,34^{\mathrm{Aa}}$ & $4,60 \pm 3,50^{\mathrm{Ba}}$ & $2,60 \pm 2,07^{\mathrm{Bb}}$ \\
\hline \multicolumn{4}{|c|}{$\checkmark \quad$ Anion gap $(6-14 \mathrm{mEq} / \mathrm{L})$} \\
\hline $\mathrm{T}_{0}$ (antes) & $8,60 \pm 1,14^{\mathrm{Aa}}$ & $7,20 \pm 1,30^{\mathrm{Bb}}$ & $7,60 \pm 1,15^{\mathrm{Bab}}$ \\
\hline $\mathrm{T}_{1}(120 \mathrm{~min})$. & $9,20 \pm 1,92^{\mathrm{Aa}}$ & $8,60 \pm 0,89^{\text {Aa }}$ & $8,80 \pm 1,30^{\mathrm{ABa}}$ \\
\hline $\mathrm{T}_{2}(410 \mathrm{~min})$. & $9,40 \pm 1,51^{\mathrm{Aa}}$ & $9,00 \pm 1,22^{\mathrm{Aa}}$ & $10,20 \pm 1,09^{\mathrm{Aa}}$ \\
\hline
\end{tabular}

${ }^{\mathrm{AB}}$ Letras maiúsculas diferentes, dentro da coluna, diferem entre si $(\mathrm{P}<0,05)$.

${ }^{a b}$ Letras minúsculas diferentes, dentro da linha, diferem entre si $(\mathrm{P}<0,05)$.

\section{Discussão}

A elevação da osmolalidade plasmática indica deficiência de água relativa aos solutos no FEC. Devido a água mover-se livremente entre o FIC e o FEC, isto também indica deficiência de água corpórea total relativa aos solutos corpóreos totais. Embora excesso de sódio possa causar hiperosmolalidade secundária à hipernatremia, a grande maioria dos casos é decorrente da excessiva perda de água associada a diminuição da ingestão de água ou excreção excessiva (Verbalis, 2003). Aparentemente, a presença de soluções dentro da porção distal do cólon menor e reto associada à distensão intestinal, bem como as alterações inflamatórias decorrentes da utilização dos enemas podem causar alteração na permeabilidade capilar e movimento de água do FEC para o lúmen intestinal (Melo et al., 2014). Outra hipótese é que tenha ocorrido um movimento de água do FEC para o lúmen intestinal na tentativa de equilibrar a osmolalidade dos dois compartimentos, embora a solução isotônica infundida tenha apresentado osmolalidade semelhante à do plasma.

A administração de diferentes soluções de enema não causou alteração nos valores séricos de ureia. Provavelmente, as soluções de enema não têm influência sobre a taxa de filtração glomerular bem como sobre a função hepática, sendo seguras sob este ponto de vista. As diferenças observadas entre os grupos dentro dos tempos são frutos da variação individual, ou do período de restrição hídrica e alimentar imposta durante a colheita dos dados. 
Durante o estudo, as concentrações de glicose diminuíram nos grupos 1 e 2, mantendo-se estável no grupo 3. A diminuição dos valores da glicose pode resultar da manipulação das amostras ou de estados de diminuição da ingestão, restrição alimentar além de outras causas (Stockham, 1995). A provável razão da discreta diminuição dos valores da glicose nos grupos 1 e 2 foi a restrição alimentar de 10 horas pela qual os animais foram submetidos durante o experimento, embora esta diminuição não tenha sido observada no grupo 3 .

O sódio é o cátion mais abundante no FEC e mudanças na sua concentração sérica geralmente refletem mudanças no equilíbrio hídrico (Seahorn \& Seahorn, 2003). Embora comuns nos pacientes em terapia intensiva, os desequilíbrios da concentração de $\mathrm{Na}^{+}$são mal compreendidos (Kraft et al., 2005). A principal mudança observada durante este estudo foi a diminuição gradual da concentração sérica de $\mathrm{Na}^{+}$no grupo 1, apesar de não caracterizar hiponatremia. Esse resultado sugere que a utilização de vários enemas em um curto período pode induzir quadros de hiponatremia.

O real mecanismo pelo qual a solução de água e sabão desencadeou diminuição significativa da concentração sérica de $\mathrm{Na}^{+}$é incerto, mas, provavelmente, a rápida infusão do enema e a permanência deste dentro da porção distal do cólon menor e reto, causando distensão, provoca desvio de fluido rico em $\mathrm{Na}^{+}$do FEC para o lúmen intestinal. Outra hipótese é que alterações inflamatórias na mucosa colônica e retal secundária a repetidos enemas provocou alterações nos mecanismos de excreção e absorção de eletrólitos e fluido neste segmento.

A repetida utilização de enemas provoca diminuição da concentração sérica de potássio. Entretanto, diferente do quadro hipocalêmico relatado na literatura (Jorgensen et al., 1985), nenhum animal desenvolveu hipocalemia com este modelo experimental.

A diminuição da concentração sérica de potássio pode resultar em diversos mecanismos, como: perda aumentada, desvio transcelular ou ingestão diminuída. A elevada perda de potássio através do trato gastrintestinal é a principal causa de diminuição dos valores séricos deste eletrólito, enquanto a diminuição da ingestão de potássio raramente ocorre (Rastergar \& Soleimani, 2001). Provavelmente, a diminuição dos valores de potássio neste estudo foi causada pela repetida administração de enemas, provocando excessiva perda de potássio junto às fezes. Embora neste modelo experimental tenham sido realizados somente três enemas é provável que a administração excessiva de enemas possa causar hipocalemia, entretanto, mais estudos são necessários para comprovar esta hipótese.

A hipocalcemia é um achado constante após a administração de enemas hipertônicos fosfatados (Jorgensen et al., 1985), e foi observada neste estudo nos animais do grupo 1. A diminuição dos valores séricos de cálcio ocorre primariamente devido à hipoalbuminemia, e para cada diminuição de $1 \mathrm{~g} / \mathrm{dL}$ na concentração de albumina (abaixo de $3 \mathrm{mg} / \mathrm{dL}$ ), a concentração sérica de cálcio total cai aproximadamente $0,8 \mathrm{mg} / \mathrm{dL}$ (Kraft et al., 2005). Entretanto, hipoalbuminemia não foi observada neste estudo. Outras causas de diminuição da concentração sérica do cálcio total são a hipomagnesemia, sepse, hiperfosfatemia, pancreatite, hipoparatireoidismo, dentre outras. Todavia, nenhum destes fatores foi identificado durante o experimento.

A administração de enemas ricos em fósforo pode provocar quadros de hipocalcemia (Jorgensen et al., 1985). Lesões ao epitélio colônico e retal após a administração de sucessivos enemas pode aumentar a absorção de fósforo e quando o produto da solubilidade cálcio-fósforo é excedido, o fósforo ligar-se ao cálcio precipitando-o. Aumentos na concentração intracelular de fosfato também promovem a captação celular do cálcio sérico, diminuindo ainda mais a concentração do cálcio (Jorgensen et al., 1985). Embora a concentração eletrolítica final das soluções administradas não tenha sido analisada, é provável que a solução de água e sabão tenha valores elevados de fósforo, entretanto isso carece de comprovação científica.

Observa-se que a concentração sérica do magnésio se manteve relativamente estável durante todo o período experimental em todos os grupos. Embora a hipomagnesemia seja comum após a administração de enemas hipertônicos (Walton et al., 2000), tal alteração não foi diagnosticada durante este estudo.

Com base nos resultados apresentados na Tabela 3 e observando a relação entre as variáveis, concluise que a administração de diferentes soluções de enema pode causar alterações no equilíbrio ácido-base. 
A solução com menor efeito sobre o equilíbrio ácido-base foi a solução eletrolítica, enquanto a solução de água com sabão provocou um quadro de alcalose metabólica.

A alcalose metabólica é caracterizada pela elevação dos valores de $\mathrm{pH}$ e $\mathrm{HCO}_{3}{ }^{-}$, ocorrendo frequentemente em associação com distúrbios do trato gastrintestinal. Muitas destas desordens estão associadas com o desenvolvimento de déficits significativos de $\mathrm{Na}^{+}$e $\mathrm{Cl}^{-}$, resultando na diminuição do volume circulante (Corley \& Marr, 1998; Durward \& Murdoch, 2003). A perda de íons hidrogênio a partir do FEC pode ocorrer secundária ao movimento de íons hidrogênio para dentro das células em resposta a diminuição da concentração de potássio (Carlson, 1997).

Neste estudo observou-se que a administração de enemas compostos de água com sabão induz ao quadro de hiponatremia, em referência ao valor de $\mathrm{T}_{0}$, e diminuição da concentração sérica de potássio. Esses dois processos associados podem desencadear um quadro de alcalose, como o observado neste grupo em associação com a perda de íons hidrogênio. Era de esperar-se que esse distúrbio fosse rapidamente compensado pela elevação da $\mathrm{pCO}_{2}$, mas isso não ocorreu. Embora os animais não tenham sido acompanhados por um período maior após o fim da administração do enema, é provável que ocorra uma resposta compensatória (Carlson, 1997).

A elevação do ânion gap em todos os grupos foi um achado inesperado. A elevação do ânion gap ocorre comumente nos quadros de acidose metabólica (Carlson, 1997; Corley \& Marr, 1998), e menos comumente nos quadros de alcalose metabólica (Bristol, 1982). A elevação do ânion gap pode refletir elevação da concentração de ânions não mensuráveis ou diminuição dos cátions não mensuráveis. Aproximadamente dois terços do ânion gap origina-se da carga negativa das proteínas séricas, enquanto o restante reflete a concentração sérica de fosfato e ânions fortes, a exemplo do lactato, sulfato, $\beta-\mathrm{OH}$ butirato, acetoacetato e ânions associados à uremia (Constable et al., 1998). Embora incomum, a alcalose metabólica desenvolvida pelos animais do grupo 1 foi acompanhada por elevação do ânion gap.

\section{Considerações Finais}

As maiores alterações observadas ocorreram no grupo da água com sabão neutro. Neste grupo, observou-se o desenvolvimento de alcalose metabólica, bem como a diminuição da concentração sérica de alguns eletrólitos. Com base nos resultados, esta solução é contraindicada em pacientes com alterações do equilíbrio ácido-base e eletrolítica, uma vez que pode exacerbar tais alterações.

\section{Referências}

Bristol, D. G. (1982). The anion gap as a prognostic indicator in horses with abdominal pain. Journal of the American Veterinary Medical Association, 181(1), 63-65. http://europepmc.org/abstract/MED/7107490

Carlson, G. P. (1997). Chapter 18 - Fluid, Electrolyte, and Acid-Base Balance. In J. J. Kaneko, J. W. Harvey, \& M. L. Bruss (Eds.), Clinical Biochemistry of Domestic Animals (Fifth Edit, pp. 485-516). Academic Press. https://doi.org/10.1016/B978-012396305-5/50019-1

Constable, P. D., Hinchcliff, K. W., \& Muir, W. W. (1998). Comparison of anion gap and strong ion gap as predictors of unmeasured strong ion concentration in plasma and serum from horses. American Journal of Veterinary Research, 59(7), 881-887. http://www.ncbi.nlm.nih.gov/pubmed/9659556

Corley, K. T. T., \& Marr, C. M. (1998). Pathophysiology, assessment and treatment of acid-base disturbances in the horse. Equine Veterinary Education, 10(5), 255-265. https://doi.org/10.1111/j.20423292.1998.tb00889.x

DiPalma, J. A., Wolff, B. G., Meagher, A., \& Cleveland, M. (2003). Comparison of reduced volume versus four liters sulfate-free electrolyte lavage solutions for colonoscopy colon cleansing. The American Journal of Gastroenterology, 98(10), 2187-2191. https://doi.org/10.1016/S00029270(03)00696-8

Durward, A., \& Murdoch, I. (2003). Understanding acid-base balance. Current Paediatrics, 13(7), 513 519. https://doi.org/10.1016/j.cupe.2003.08.009

Edwards, G. B. (1997). Diseases and Surgery of the Small Colon. Veterinary Clinics of North America: Equine Practice, 13(2), 359-375. https://doi.org/10.1016/S0749-0739(17)30245-6

Ferreira, C., Palhares, M. S., Melo, U. P., Gheller, V. A., \& Braga, C. E. (2009). Cólicas por 
compactação em equinos: etiopatogenia, diagnóstico e tratamento. Acta Veterinaria Brasilica, 3(3), $117-126$.

Geovú, L. B., Costa, A. P. D., Carvalho, C. B., Detmann, E., \& Carvalho, C. S. P. (2006). Osmolalidade plasmática e osmol gap em eqüinos desidratados experimentalmente. Arquivo Brasileiro de Medicina Veterinária e Zootecnia, 58(5), 744-748. https://doi.org/10.1590/S0102-09352006000500007

Jorgensen, L. S., Center, S. A., Randolph, J. F., \& Brum, D. (1985). Electrolyte abnormalities induced by hypertonic phosphate enemas in two cats. Journal of the American Veterinary Medical Association, 187(12), 1367-1368. http://europepmc.org/abstract/MED/4086355

Kraft, M. D., Btaiche, I. F., Sacks, G. S., \& Kudsk, K. A. (2005). Treatment of electrolyte disorders in adult patients in the intensive care unit. American Journal of Health-System Pharmacy, 62(16), 1663-1682. https://doi.org/10.2146/ajhp040300

Melo, U. P., Ferreira, C., \& Palhares, M. S. (2007). Motilidade gastrointestinal equina: fisiologia, mecanismos de disfunção e efeito da administração de diferentes drogas. Rev. CFMV , 41, 41-56.

Melo, U. P., Palhares, M. S., Ferreira, C., Evaristo, I. G. B., Leme, F. O. P., Serakides, R., \& Silva Filho, J. M. (2008). Efeito de diferentes soluções de enema sobre os parâmetros clínicos de eqüinos. Arquivo Brasileiro de Medicina Veterinária e Zootecnia, 60(3), 525-535. https://doi.org/10.1590/S010209352008000300002

Melo, U. P., Palhares, M. S., Ferreira, C., França, S. A., Serakides, R., \& Silva, F. (2014). The effect that different enema solutions may have on macro and microscopic characteristics of the mucosa of the colon and rectum in horses. A Hora Veterinária, 34(201), 43-49.

Rastergar, A., \& Soleimani, M. (2001). Hypokalaemia and hyperkalaemia. Postgraduate Medical Journal, 77(914), 759 LP - 764. https://doi.org/10.1136/pgmj.77.914.759

SAS. (2004). SAS/STAT User guide, Version 9.1.2. SAS Institute Inc.

Schmelzer, M., Schiller, L. R., Meyer, R., Rugari, S. M., \& Case, P. (2004). Safety and effectiveness of largevolume enema solutions. Applied Nursing Research, 17(4), 265-274. https://doi.org/10.1016/j.apnr.2004.09.010

Seahorn, J. L., \& Seahorn, T. L. (2003). Fluid therapy in horses with gastrointestinal disease. Veterinary Clinics of North America: Equine Practice, 19(3), 665-679. https://doi.org/10.1016/j.cveq.2003.08.005

Stockham, S. L. (1995). Interpretation of Equine Serum Biochemical Profile Results. Veterinary Clinics of North America: Equine Practice, 11(3), 391-414. https://doi.org/10.1016/S0749-0739(17)30307-3

Verbalis, J. G. (2003). Disorders of body water homeostasis. Best Practice \& Research Clinical Endocrinology \& Metabolism, 17(4), 471-503. https://doi.org/10.1016/S1521-690X(03)00049-6

Walton, D. M., Thomas, D. C., Aly, H. Z., \& Short, B. L. (2000). Morbid Hypocalcemia Associated With Phosphate Enema in a Six-Week-Old Infant. PEDIATRICS, 106(3), e37-e37. https://doi.org/10.1542/peds.106.3.e37

Histórico do artigo:

Recebido: 1 de setembro de 2020.

Aprovado: 4 de dezembro de 2020 .

Disponível online: 4 de março de 2021
Licenciamento: Este artigo é publicado na modalidade Acesso Aberto sob a licença Creative Commons Atribuição 4.0 (CC-BY 4.0), a qual permite uso irrestrito, distribuição, reprodução em qualquer meio, desde que o autor e a fonte sejam devidamente creditados. 